\title{
Evaluación del uso de herramientas sincrónicas y asincrónicas en procesos de formación de las ciencias agropecuarias
}

\section{Synchronous and asynchronous tools use evaluation in the agricultural sciences training processes}

Fecha recepción: 14 de julio de 2015

Fecha aceptación: 1 de septiembre de 2015

Edwin Manuel Páez-Barón ${ }^{1}$

Emma Sofía Corredor-Camargo ${ }^{2}$ Jorge Armando Fonseca-Carreño ${ }^{3}$

\begin{abstract}
Resumen
La educación a distancia ha tenido un gran avance durante los últimos años, gracias a la incorporación de las herramientas que proporcionan las Tecnologías de la información y la comunicación -TIC-. Estas herramientas pueden ser sincrónicas y asincrónicas; con las primeras, los interlocutores se conectan al mismo tiempo y establecen procesos de comunicación en tiempo real; las segundas permiten la interacción y comunicación en diferentes tiempos, por lo cual no se requiere la participación de los interlocutores al mismo tiempo. Se evaluó el uso de estas herramientas en seis cursos de las Ciencias Agropecuarias de la Universidad Nacional Abierta y a Distancia -UNAD-. Los resultados permitieron evidenciar que las herramientas sincrónicas son utilizadas en menor medida, a pesar de que existe mayor preferencia hacia su uso (55.7\%) y una percepción en los estudiantes acerca de que facilitan mayor interacción con el docente y los otros compañeros. Su principal limitante se encuentra en la exigencia de un horario específico de conexión para la interacción y, en algunos casos, en las dificultades en la conectividad.
\end{abstract}

Palabras clave: aprendizaje; blended-learning; educación a distancia; e-learning; mobile-learning.

\begin{abstract}
Distance education has achieved a great advance in recent years, thanks to the Information and Communications Technology -ICTs- tools incorporation. These communication tools can be classified as synchronous and asynchronous, the first ones are those in which their users' groups are interconnected at

1 Ph. D. Universidad Nacional Abierta y a Distancia (Tunja-Colombia). edwin.paez@unad.edu.co.

2 M. Sc.. Universidad Nacional Abierta y a Distancia (Tunja-Colombia). emma.corredor@unad.edu.co.

3 M. Sc. Universidad Nacional Abierta y a Distancia (Tunja-Colombia). jorge.fonseca@unad.edu.co.
\end{abstract}


the same time and establish a communication processes in real time, the second ones allow interaction and communication at different moments, so the participation of the partners is not required to be at the same time.

These tools use in six courses of agricultural sciences of the Universidad Nacional Abierta y a Distancia -UNAD- was evaluated. The results demonstrated that the use of synchronous tools is low, although students prefer them $(55.7 \%)$, and think they facilitate a greater interaction with their faculty and peers. Their main limitation is the requirement to be at a specific timetable for their interaction and connection, besides that in some cases due to connectivity difficulties.

Keywords: blended-learning; distance education; e-learning; learning; mobile-learning. 


\section{Introducción}

Durante los últimos años, la educación ha tenido un gran desarrollo, permitiendo la incorporación de múltiples herramientas tecnológicas en los procesos pedagógicos y de formación. La educación en las ciencias agropecuarias no es ajena a esta realidad, $y$, como tal, ha estado enmarcada en un proceso de avance y de incorporación de nuevas herramientas pedagógicas; esta dinámica es más evidente en los procesos de formación a distancia, que, gracias al avance tecnológico, registran un gran desarrollo, llegando a constituirse en una alternativa de calidad y con procesos pedagógicos que eliminan esas barreras físicas que limitaban su expansión. En este sentido, las Tecnologías de la Información y la comunicación - TIC- se han convertido en un elemento fundamental en los procesos formativos desarrollados a nivel de los ambientes virtuales de aprendizaje (1), que ya no solo hacen parte de los procesos de educación a distancia, sino también de los de educación tradicional, que han visto la necesidad de acoplarse a la nueva realidad educativa de un mundo cambiante y en permanente evolución; por ello, en la actualidad existen diversos métodos de educación que incorporan las herramientas brindadas por las TIC para el desarrollo de los procesos formativos, como son el Blended-Learning, el E-Learning y el MobileLearning, que incorporan y usan intensivamente estas herramientas $(2,3,4)$.

Hasta hace unos años, la educación a distancia se limitaba a unos procesos de transmisión de conocimientos de los docentes a los estudiantes, utilizando medios de comunicación que podían tardar varios días en llegar al receptor; se constituía, básicamente, en un modelo de comunicación unidireccional con poca o nula interacción y basado en el desarrollo de unas tareas específicas. Actualmente, los procesos de comunicación en los modelos de educación a distancia se realizan prácticamente de forma inmediata $y$ en tiempo real, permitiendo el desarrollo de un proceso formativo más dinámico y participativo en donde intervienen múltiples actores: el docente, los estudiantes y la comunidad donde se localizan, entre otros. Este proceso dinámico se facilita gracias a la incorporación de múltiples herramientas comunicativas en los ambientes de formación, algunas de las cuales permiten la comunicación sincrónica, o en tiempo real, como el chat o la videoconferencia, desarrollada con diferentes aplicaciones; o la comunicación de tipo asincrónico, donde los actores involucrados pueden comunicarse en diferentes tiempos, como en los foros y el correo electrónico.

El auge tecnológico, y en particular la internet, ha Ilevado a que los seres humanos estén desarrollando constantemente procesos de comunicación; vivimos hoy en una 'gran aldea global', en donde prácticamente ya no existen barreras geográficas o de tiempo que limiten la comunicación con otras personas localizadas a grandes distancias; basta con hacer un clic para acceder a una red que permite interconectar a personas en todo el mundo. Lo anterior ha contribuido a que la educación llegue a lugares donde anteriormente era prácticamente imposible que Ilegara; hoy la educación, en sus diferentes niveles, es asequible en la gran mayoría de territorios. Como lo señalan Cabero y Llorente (3), la red ha dejado de ser un entorno tecnológico para convertirse en uno social, con participación e interacción constante, y, en este sentido, se está convirtiendo en un entorno público y globalizado, por lo cual es posible aprovechar las herramientas que ofrece para su incorporación en los procesos de formación a nivel de la educación superior.

\section{Materiales y métodos}

El objetivo de la investigación fue evaluar el uso de herramientas sincrónicas y asincrónicas en los procesos de aprendizaje a nivel de entornos virtuales en seis cursos de las ciencias agropecuarias de la Universidad Nacional Abierta y a Distancia UNAD-; con ello se buscó identificar la preferencia de los estudiantes hacia el uso de herramientas sincrónicas o asincrónicas y caracterizar el uso de estas en los procesos formativos en ambientes virtuales de aprendizaje. Se desarrolló un estudio de tipo descriptivo cualitativo para determinar el uso de herramientas sincrónicas y asincrónicas, así como la percepción de las ventajas y desventajas en los cursos evaluados: para esto se aplicó un 
instrumento tipo encuesta en un formato digital de registro automático de las respuestas, el cual permitió conocer el grado de uso y preferencia de los estudiantes frente a estas herramientas. La población objeto de estudio estuvo conformada por un total de 360 estudiantes, y se tomó una muestra de 70 de ellos, los cuales respondieron la encuesta de forma voluntaria en línea. Los estudiantes estaban localizados en diferentes zonas del país, ya que la oferta de los cursos evaluados es de orden nacional. Los cursos virtuales evaluados se desarrollan bajo la plataforma Moodle, una plataforma dinámica de uso libre para la creación de entornos virtuales de aprendizaje. Las herramientas sincrónicas evaluadas fueron:

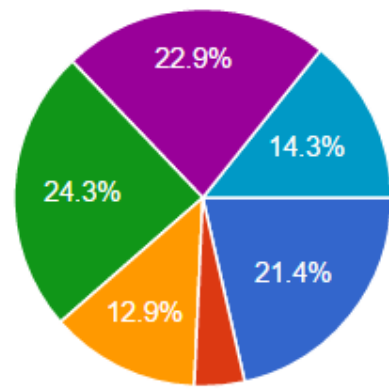

Skype, chat en línea y telefonía fija o celular; las herramientas asincrónicas fueron: correo interno, foros de contacto e interacción.

Se evaluaron aspectos de preferencia de las herramientas para comunicación con el docente, con los otros estudiantes, los horarios de uso y las causas de uso y no uso de las diversas herramientas.

\section{Resultados y discusión}

Los cursos evaluados fueron seis: cuatro correspondientes a programas de pregrado en Zootecnia y Agronomía, y dos, a programas posgraduales en el área de Especialización en Nutrición Animal Sostenible (Figura 1).

$\begin{array}{rrr}\text { Reproducción Avanzada } & \mathbf{1 5} & 21.4 \% \\ \text { Salud Animal } & \mathbf{3} & 4.3 \% \\ \text { Sistema Ecológico Etológico } & \mathbf{9} & 12.9 \% \\ \text { Agricultura Biológica } & \mathbf{1 7} & 24.3 \% \\ \text { Teoría General de Sistemas } & \mathbf{1 6} & 22.9 \% \\ \text { Mejoramiento Animal } & \mathbf{1 0} & 14.3 \%\end{array}$

Figura 1. Cursos evaluados en el proyecto.

La caracterización de la población encuestada permitió determinar que 53 estudiantes, correspondiente al $75.7 \%$ de la población encuestada, viven en zonas urbanas, y 17 (24.3\%), en zona rural (Figura 2). Estos datos están muy relacionados con los porcentajes de población localizada en las zonas rurales en Colombia, que, de acuerdo con datos del Banco Mundial, corresponde al $24 \%$ del total de la población del país (5). En este aspecto es importante mencionar que la educación a distancia puede constituirse en una oportunidad real de acceso a la educación superior para aquellas poblaciones localizadas en zonas geográficas distantes, donde por diversas razones (personales, laborales, económicas, etc.) no es posible acceder a los modelos tradicionales de educación, por lo cual, la educación a distancia, y las diferentes herramientas pedagógicas y de comunicación que incorpora, pueden permitirle el desarrollo de sus procesos formativos con calidad en cualquier tiempo y lugar. 


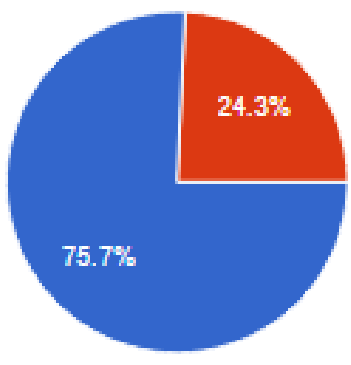

\section{Zona Urbana $\quad 53 \quad 75.7 \%$ \\ Zona Rural $17 \quad 24.3 \%$}

Figura 2. Lugar de vivienda de los encuestados.

En los datos que se brindan a continuación, el estudiante tuvo la opción de seleccionar más de una respuesta, por lo cual los valores y el porcentaje de ellos para cada una de las opciones se indican con base en el número total de la muestra evaluada (correspondiente a 70 estudiantes). Respecto al lugar de acceso en donde el estudiante desarrolla habitualmente las actividades académicas de los cursos (Figura 3), no existe preferencia por un único lugar; en tal sentido, el estudiante realiza el acceso desde diferentes ubicaciones durante el desarrollo de su proceso formativo. La mayoría lo realiza desde su lugar de vivienda (65 estudiantes, correspondiente al $92.9 \%$ del total); una menor proporción, en otros sitios, como el lugar de trabajo (23 estudiantes, correspondientes al 32.9 $\%$ del total de la muestra) o locales comerciales (21 estudiantes, correspondientes a un 30\% del total de la muestra), y un número reducido lo realiza directamente en instalaciones de la Universidad (6 estudiantes, correspondientes a un $8.6 \%$ del total de la muestra). El acceso desde instalaciones de la universidad es muy bajo, lo cual puede estar representado principalmente por los costos que implica el desplazamiento hasta el sitio de ubicación de los diferentes centros de la universidad, sumado a la gran ventaja de poder contar con el acceso a las aulas virtuales desde cualquier lugar de la geografía mundial que cuente con acceso a internet.

$\begin{array}{rrr}\text { Casa u Hogar. } & 65 & 92.9 \% \\ \text { Lugar de Trabajo. } & \mathbf{2 3} & 32.9 \% \\ \text { Café Internet o local comercial. } & \mathbf{2 1} & 30 \% \\ \text { Universidad. } & 6 & 8.6 \% \\ \text { Otro } & \mathbf{2} & 2.9 \%\end{array}$

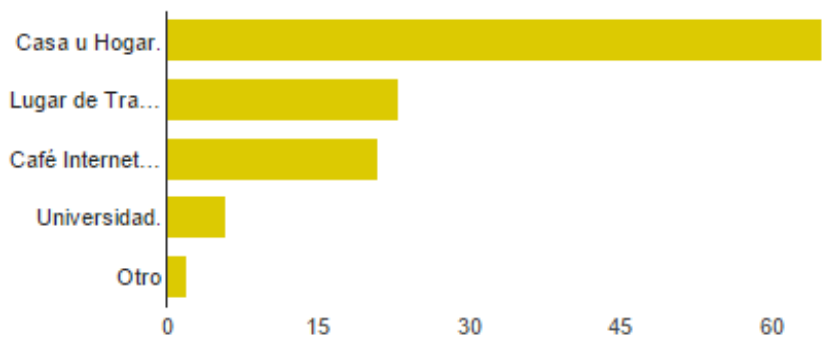

Figura 3. Lugar de acceso a los cursos virtuales.

Con referencia al tipo de conexión a internet utilizado para el acceso a los cursos virtuales (Figura 4), existe también variación; en general, se utiliza más de un tipo de conexión para ese acceso a las aulas virtuales. El estudiante tuvo la opción de dar más de una respuesta, por lo cual los valores y el porcentaje para cada una de las opciones se indican con base en el número total de la muestra evaluada (correspondiente a 70 estudiantes). La mayoría utiliza conexiones de tipo fijo o banda ancha (44 estudiantes, correspondientes al 62.9 $\%$ del total de la muestra); 31 estudiantes (44.3\% del total) usan el internet de tipo móvil, a través de módem; 18 estudiantes (25.7\%), los planes de datos móviles, y 13 estudiantes (18.6\%), internet de tipo satelital. Lo anterior denota un acceso elevado 
de los estudiantes a redes de conexión fija que, por lo general, tienen buenos niveles de estabilidad y conectividad, que facilitan el acceso y el desarrollo de las actividades de trabajo y comunicación en los ambientes virtuales. Sin embargo, existe también un amplio uso de conexiones móviles que facilitan el acceso en cualquier ubicación geográfica; este tipo de conexiones ha tenido un gran aumento en cobertura durante los últimos años.
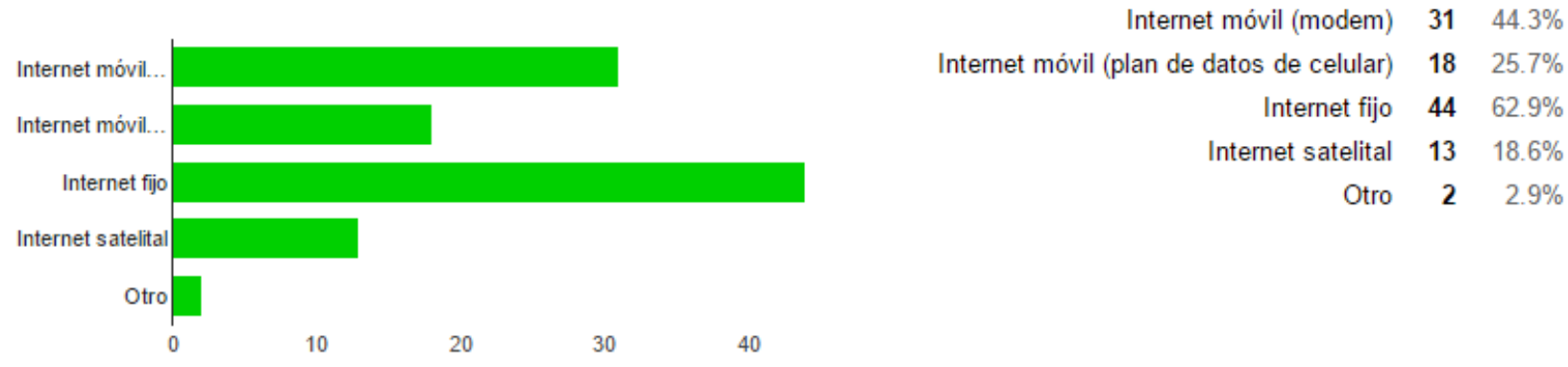

Figura 4. Tipo de acceso a internet.

\section{Utilización de las herramientas de comunicaciones sincrónicas y asincrónicas}

Respecto a la utilización de las herramientas de comunicación, se observa que existe mayor utilización de herramientas de tipo asincrónico en los cursos evaluados; sin embargo, la preferencia del estudiante es hacia el uso de herramientas sincrónicas $(55.7 \%$ del total de los encuestados las prefieren; ver Figura 5), pero existen algunas limitantes que hacen que su uso sea menor a nivel de los cursos virtuales.

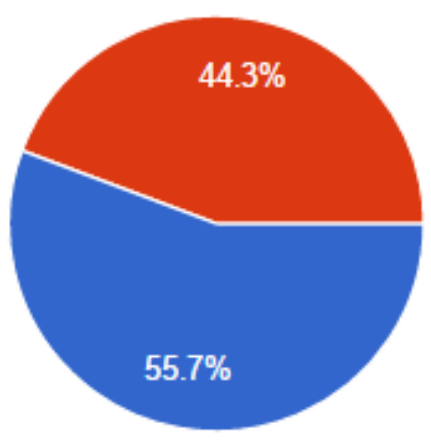

\section{Sincrónicas $\quad 39 \quad 55.7 \%$ Asincrónicas $\quad 31 \quad 44.3 \%$}

Figura 5. Preferencia de uso herramientas sincrónicas vs. asincrónicas.

\section{Contacto de los estudiantes con el docente}

Para el contacto, la interacción y la comunicación del estudiante con el docente se prefiere el uso de los foros de trabajo (Figura 6); es importante señalar que estos son una herramienta que favorece la interacción y construcción colectiva del conocimiento, bajo la guía y el direccionamiento del docente, pero con la autonomía y el liderazgo del estudiante como artífice de su propio conocimiento (6). El $81.4 \%$ de los estudiantes hacen uso regular de los foros de trabajo para contactar al docente: el $45.7 \%$ los utilizan siempre, y el $35.7 \%$, casi siempre (Figura 6). En segundo lugar de preferencia está el correo interno de los cursos, con el $70 \%$ de utilización por los estudiantes: $38.6 \%$ lo utilizan siempre, y $31.4 \%$, casi siempre (Figura 7); esta es una herramienta de tipo asincrónico que permite el contacto directo con el docente, facilitando el intercambio de información con los diferentes actores del curso, sin embargo, su uso es bajo frente al potencial que 
representa. En tercer lugar de preferencia están los foros de contacto; solo el $52.9 \%$ de los estudiantes utilizan con frecuencia este medio (el $28.6 \%$ lo utilizan siempre, y el $24.3 \%$, casi siempre); el $21.4 \%$ lo utilizan algunas veces; el $15.7 \%$, casi nunca, y el $10 \%$ nunca lo utilizan para el contacto con el docente (Figura 8).

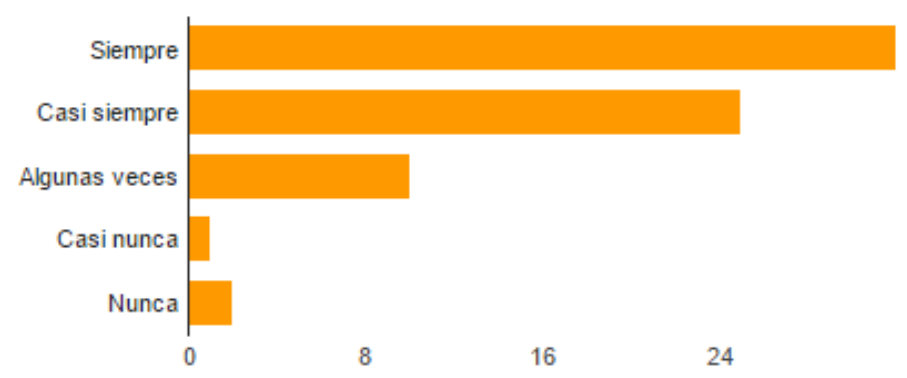

$$
\begin{array}{rrr}
\text { Siempre } & \mathbf{3 2} & 45.7 \% \\
\text { Casi siempre } & \mathbf{2 5} & 35.7 \% \\
\text { Algunas veces } & \mathbf{1 0} & 14.3 \% \\
\text { Casi nunca } & \mathbf{1} & 1.4 \% \\
\text { Nunca } & \mathbf{2} & 2.9 \%
\end{array}
$$

Figura 6. Uso de foros de trabajo para contacto con docente (colaborativo y práctico.)

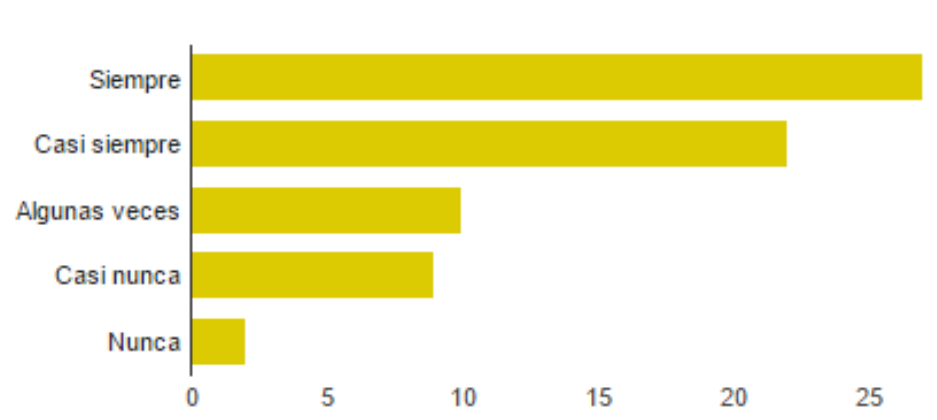

$\begin{array}{rrr}\text { Siempre } & \mathbf{2 7} & 38.6 \% \\ \text { Casi siempre } & \mathbf{2 2} & 31.4 \% \\ \text { Algunas veces } & \mathbf{1 0} & 14.3 \% \\ \text { Casi nunca } & \mathbf{9} & 12.9 \% \\ \text { Nunca } & \mathbf{2} & 2.9 \%\end{array}$

Figura 7. Uso de correo interno para contacto con docente.

Respecto a los foros de contacto, se diferencian dos tipos: un foro de novedades, donde el docente publica noticias o información de carácter informativo durante el desarrollo del curso, y un foro general, en donde el estudiante intercambia ideas y opiniones y realiza sus aportes en la temática del curso. Estos foros también son un espacio fundamental para la interacción y permiten potenciar el proceso participativo en los ambientes virtuales de aprendizaje, pero tampoco han sido aprovechados al máximo.

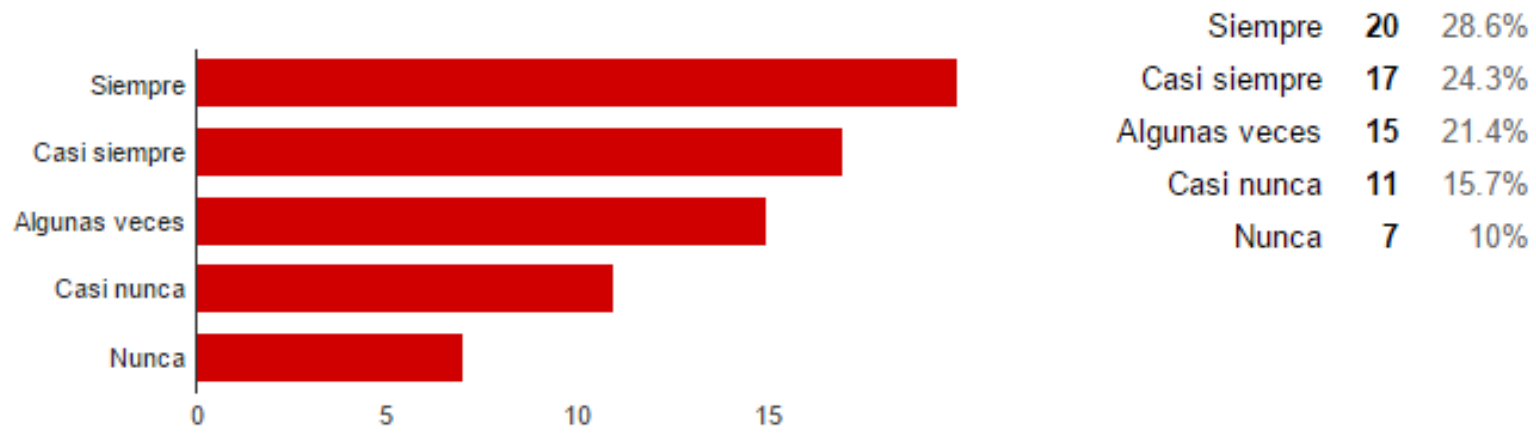

Figura 8. Uso de foros de contacto (novedades y general) para contacto con docente. 


\section{Contacto de los estudiantes con los compañeros}

Para el contacto con los otros estudiantes se prefiere el uso de los foros de trabajo, el teléfono celular y, en menor medida, el correo. Los foros de trabajo, de tipo colaborativo o práctico, son una herramienta de comunicación asincrónica de gran utilidad y son utilizados por el $88.5 \%$ de los estudiantes (51.4\%, siempre, y $37.1 \%$, casi siempre) (Figura 9). Es importante destacar que en este tipo de foros es donde se realiza la mayor parte de la interacción de los estudiantes para dar lugar al desarrollo de las actividades formativas del curso. Los datos denotan buena utilización de estos espacios, los cuales constituyen el punto de encuentro para el desarrollo de las actividades colaborativas, favoreciendo el intercambio de ideas y aportes para el desarrollo de los productos del curso y la construcción participativa del conocimiento.

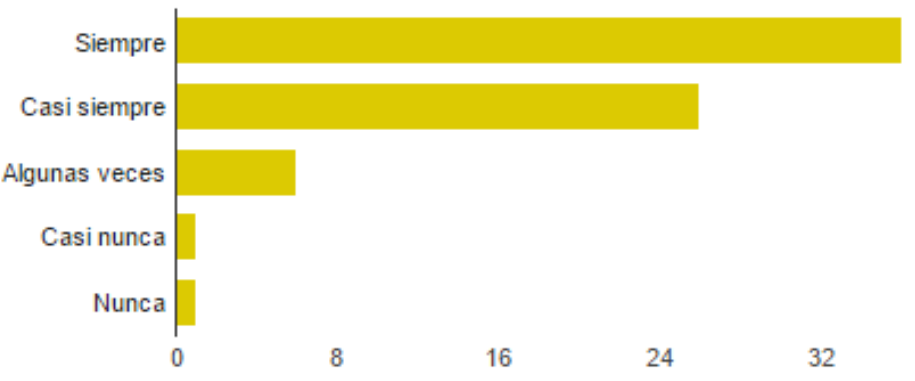

$\begin{array}{rrr}\text { Siempre } & \mathbf{3 6} & 51.4 \% \\ \text { Casi siempre } & \mathbf{2 6} & 37.1 \% \\ \text { Algunas veces } & \mathbf{6} & 8.6 \% \\ \text { Casi nunca } & \mathbf{1} & 1.4 \% \\ \text { Nunca } & \mathbf{1} & 1.4 \%\end{array}$

Figura 9. Uso de foros de trabajo para contacto con estudiantes (colaborativo y práctico).

Los foros de contacto (foro de novedades y foro general) son utilizados en menor medida, encontrándose que el $50 \%$ de los estudiantes los usan con frecuencia $(22.9 \%$, siempre, y $27.1 \%$, casi siempre); el $17.1 \%$, algunas veces; el 24.3 $\%$, casi nunca, y el $8.6 \%$ nunca han utilizado esta herramienta. Estos datos indican que un alto porcentaje no utiliza esta herramienta asincrónica, que es de gran utilidad para la interacción con los otros estudiantes y puede contribuir de gran manera en los procesos formativos de los ambientes virtuales de aprendizaje.

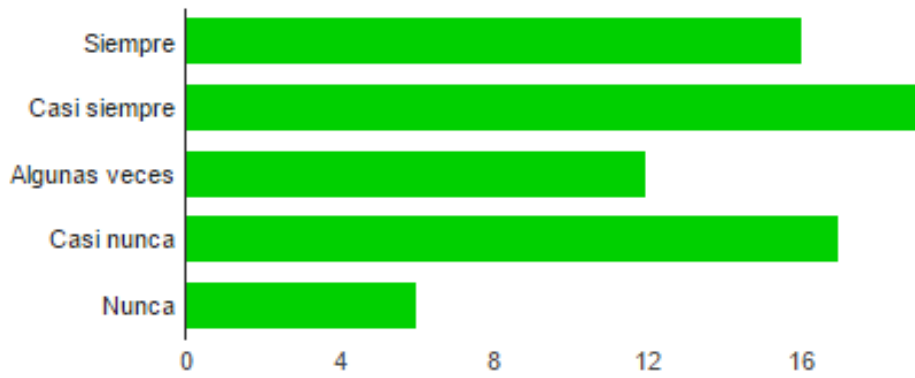

$\begin{array}{rrr}\text { Siempre } & \mathbf{1 6} & 22.9 \% \\ \text { Casi siempre } & \mathbf{1 9} & 27.1 \% \\ \text { Algunas veces } & \mathbf{1 2} & 17.1 \% \\ \text { Casi nunca } & \mathbf{1 7} & 24.3 \% \\ \text { Nunca } & \mathbf{6} & 8.6 \%\end{array}$

Figura 10. Uso de foros de contacto (novedades y general).

El Skype tiene un nivel medio de utilización; se observa que el $38.6 \%$ de los estudiantes utilizan esta herramienta con frecuencia para el contacto con los otros estudiantes (el $22.9 \%$, siempre, y el $15.7 \%$, utilizan casi siempre); el $17.1 \%$, algunas veces; el $12.9 \%$, casi nunca, y el $31.4 \%$, nunca
(Figura 10). Esta es una herramienta sincrónica que permite la comunicación en tiempo real por tres formas: chat escrito, audio o video, siendo de gran utilidad para el contacto directo, por lo cual se debe propugnar un mejor aprovechamiento de ella. 


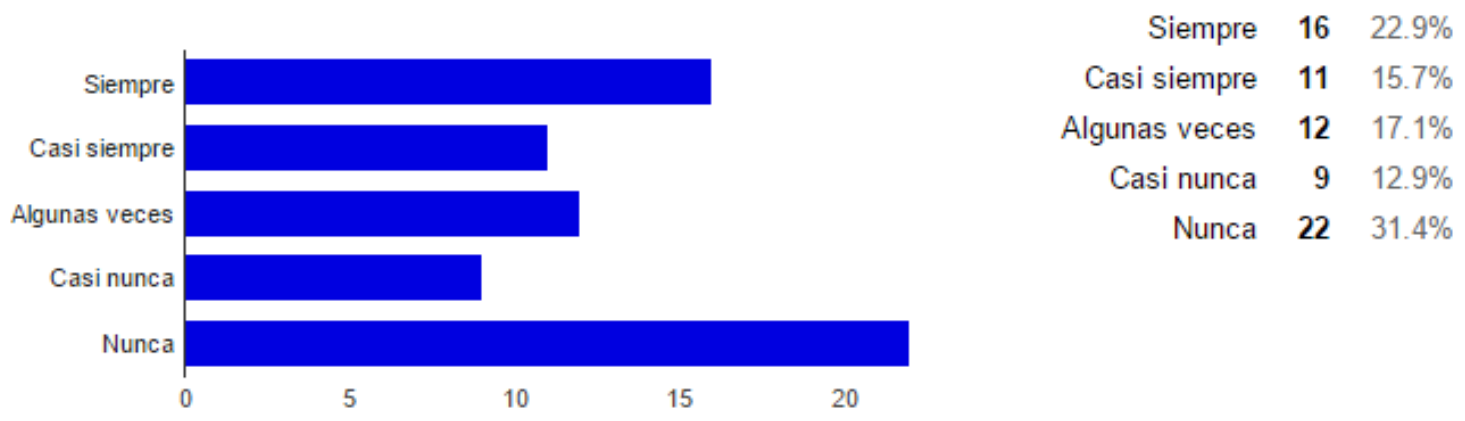

Figura 11. Uso de Skype para contacto con otros estudiantes.

Respecto al uso de telefonía fija o celular, el 48.6 $\%$ de los estudiantes hacen uso frecuente de ella (30\%, siempre, y $18.6 \%$, casi siempre); el $30 \%$ la utilizan algunas veces; el $8.6 \%$, casi nunca, y el $12.9 \%$, nunca (Figura 11). En este sentido, es importante señalar que también se debe alentar el incremento del uso de esta herramienta de comunicación sincrónica, teniendo en cuenta que la telefonía móvil en Colombia alcanzó, en el 2014, los 55,3 millones de líneas activas y un índice de penetración de $116 \%$, según el informe trimestral del Ministerio TIC (7), razón por la cual es una herramienta con la que cuentan la mayoría de los estudiantes; en consecuencia, debe aprovecharse y motivarse hacia su uso pedagógico en los procesos formativos. Esta herramienta se ha utilizado en los cursos para, principalmente, establecer acuerdos acerca de la dinámica de trabajo para el desarrollo de las diversas actividades de los cursos virtuales.

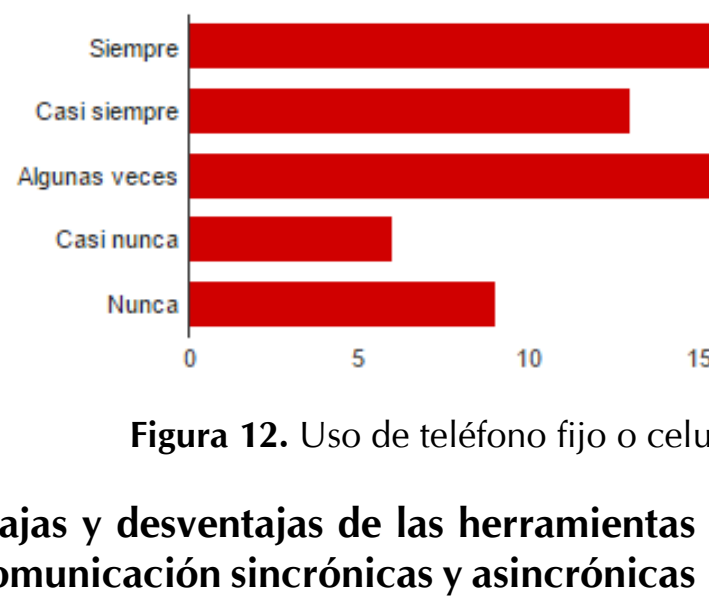

Existen muchas ventajas en el uso de estas herramientas de comunicación en los procesos académicos; sin embargo, es importante señalar que a nivel formativo deben estar articuladas con las estrategias de aprendizaje para que su utilidad realmente contribuya al proceso de formación y no sean solo un elemento o accesorio más en los cursos. En los datos que se brindan a continuación, el estudiante tuvo la opción de seleccionar más de una respuesta, por lo cual los valores y el porcentaje de estos para cada una de las opciones se indican con base en el número total de la muestra evaluada (correspondiente a 70 estudiantes).

En referencia al Skype, la población encuestada reconoce diferentes ventajas; se manifiesta que una de las principales está representada por la interacción que se da con el docente (58 estudiantes, correspondientes al $82.9 \%$ del total) y por la interacción que se puede desarrollar con los demás estudiantes (48 estudiantes, correspondientes al $68.6 \%$ ). Se reconocen otras ventajas, tales como la facilidad de uso (45 
estudiantes, correspondientes al $64.3 \%$ del total) y la respuesta inmediata que se brinda por medio de esta herramienta (35 estudiantes, correspondientes al $50 \%$ del total). En menor medida se señalan como ventajas los horarios de disponibilidad de esta herramienta (15 estudiantes, correspondientes al $21.4 \%$ del total), la cual está disponible todo el tiempo, y la facilidad de conexión (26 estudiantes, correspondientes al $37.1 \%$ del total) (Figura 12). El Skype es una herramienta de gran utilidad que puede contribuir a mejorar las competencias comunicativas orales y digitales de los estudiantes (8), por lo cual puede representar un plus en los procesos de formación en ambientes virtuales.
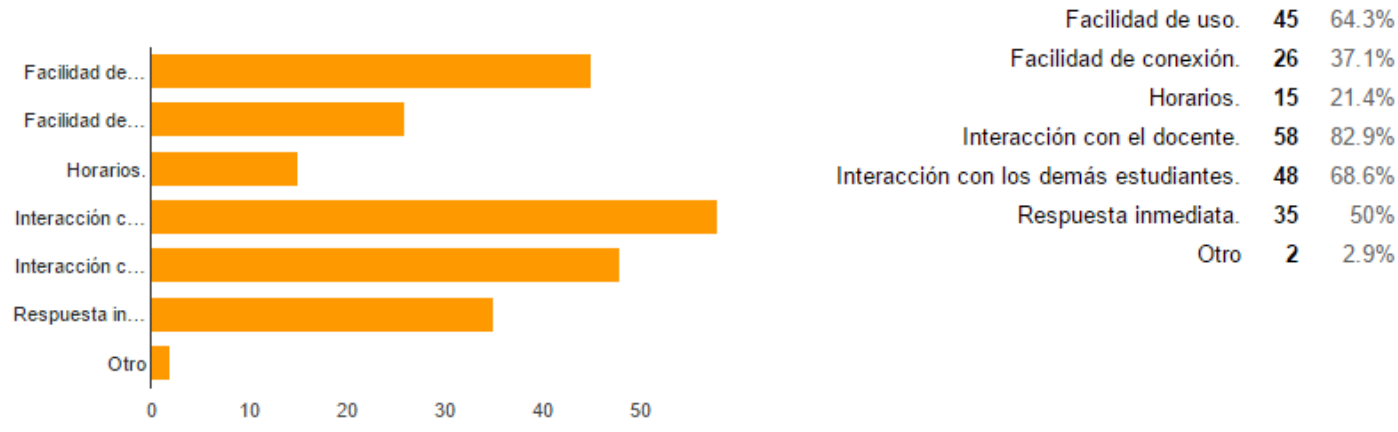

Figura 13. Ventajas del uso del Skype.

Respecto a las desventajas del uso del Skype, 42 estudiantes (correspondientes al $60 \%$ del total) reconocen que la principal son los horarios, puesto que en los cursos se brinda un horario específico para desarrollar los procesos de comunicación con Skype; otra de las desventajas planteadas es la dificultad de conexión (24 estudiantes, correspondientes al $34.3 \%$ del total) (Figura 13), relacionada en algunos casos con la necesidad de una línea de internet de alta velocidad (banda ancha o móvil de alta velocidad).
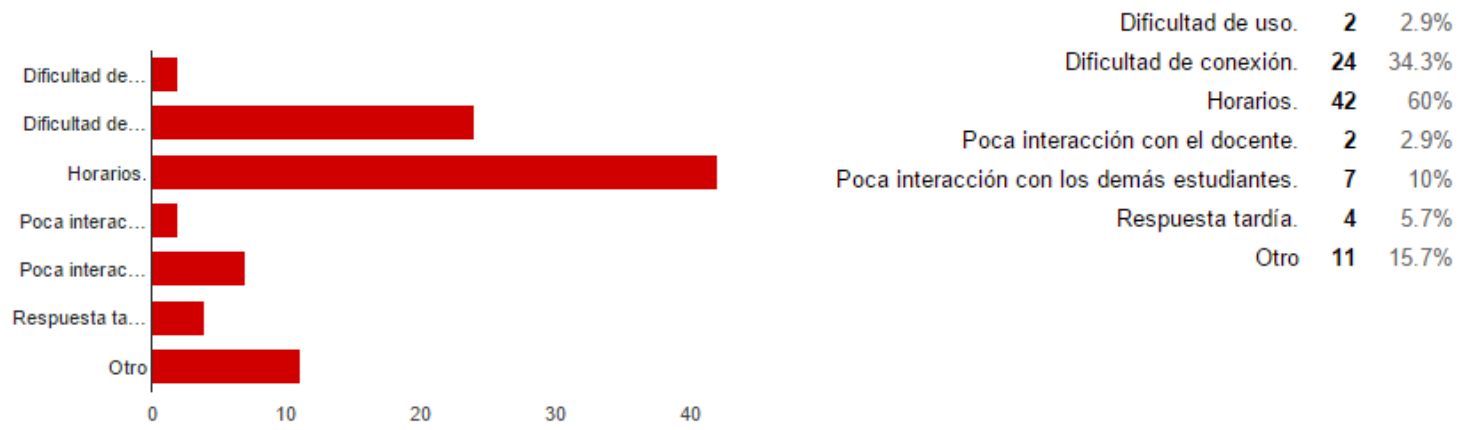

Figura 14. Desventajas del uso del Skype.

En referencia a las ventajas de los foros de trabajo (colaborativo y práctico), los estudiantes reconocen que son amplias, y como tal se identifican con varias de ellas, entre las cuales se destacan los horarios (37 estudiantes, correspondientes al $52.9 \%$ del total), lo cual puede explicarse por la facilidad de establecer contacto en cualquier momento, ya que se trata de una herramienta asincrónica. Otra ventaja señalada es la facilidad de uso (52 estudiantes, correspondientes al 74.3 $\%$ del total), ya que su manejo es similar al uso de un correo electrónico; asimismo, se menciona la posibilidad de interacción con el docente (53 estudiantes, correspondientes al $75.7 \%$ del total), siendo una herramienta de comunicación bidireccional asincrónica, y también se reconoce 
como ventaja la posibilidad de interación con

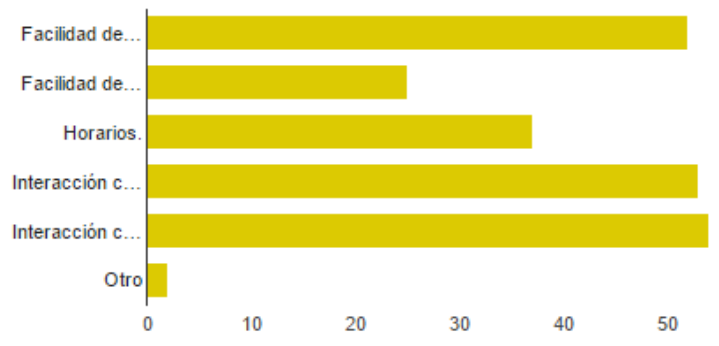

los otros estudiantes del curso (54 estudiantes, correspondientes al $77.1 \%$ del total) (Figura 13).

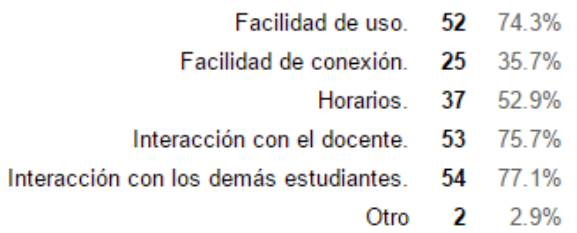

Figura 15. Ventajas del uso de los foros de trabajo.

Respecto a las desventajas de los foros de trabajo (colaborativo y práctico), los estudiantes relacionan la respuesta tardía (29 estudiantes, correspondientes al $41.4 \%$ del total), que se puede explicar por su característica de herramienta asincrónica, por lo cual el interlocutor puede ingresar en cualquier momento a brindar aportes o respuestas; sin embargo, es importante señalar que, para el caso de los docentes, la respuesta es brindada, en la mayoría de los casos, en un período inferior a 24 horas. Otra de las desventajas señaladas son las dificultades en la conexión (19 estudiantes,

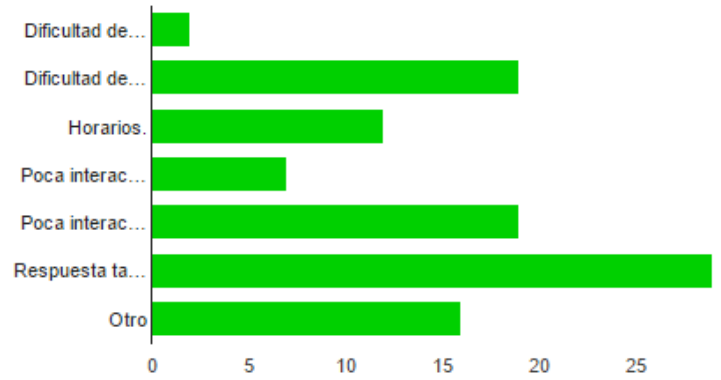

correspondientes al $27.1 \%$ del total), pero esta depende más de la velocidad y la conexión específica que se establece por el estudiante (Figura 14); igualmente, la poca interacción con los demás estudiantes se indica como una desventaja, aquí es necesario señalar que el docente juega un papel fundamental para estimular la participación y el aporte de los estudiantes, pero estos, como seres autónomos son quienes deciden el tiempo y la cantidad de aportes o participaciones realizadas, por lo cual la responsabilidad juega un papel primordial en el éxito de estas interacciones.

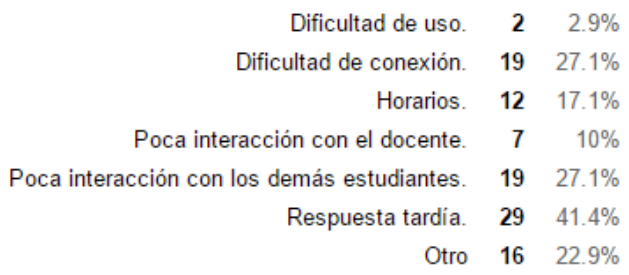

Figura 16. Desventajas del uso de los foros de trabajo.

En cuanto al chat en línea, existen diversas ventajas; la principal, según la mayoría de los estudiantes (56 estudiantes, correspondientes al $80 \%$ del total), es la interacción con el docente; también se reconocen como ventajas la interacción con los demás estudiantes (38 estudiantes, correspondientes al $54.3 \%$ del total) y la respuesta inmediata que ofrece el uso de esta herramienta (31 estudiantes, correspondientes a un $44.3 \%$ del total) (Figura 15); su característica de sincronía permite un contacto directo y en tiempo real que mejora la interacción y el intercambio de ideas y opiniones. Es importante señalar que, como lo menciona Verdugo (9), el chat es una herramienta muy útil, pero que ha sido poco explotada en los procesos de formación, y puede brindar muchas utilidades al ser incorporada dentro de los procesos formativos, especialmente para el desarrollo de 
habilidades de escritura y argumentación; sin embargo, es importante contar con grupos que no sean tan numerosos, con el fin de permitir una mejor interacción y un debate más organizado y productivo (10).
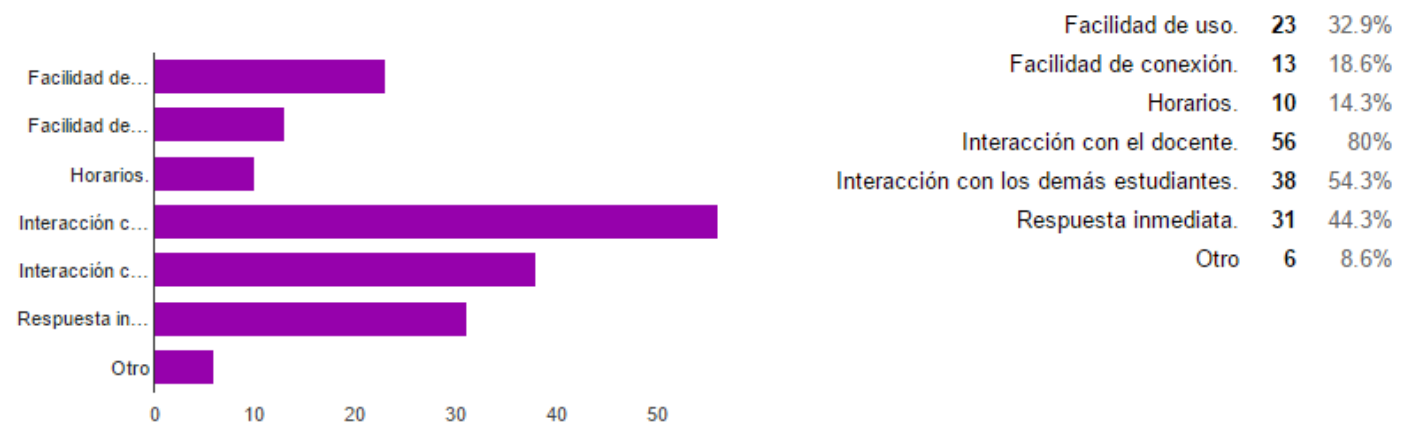

Figura 17. Ventajas del uso del Chat en línea.

En referencia a las desventajas del chat en línea, 38 estudiantes (correspondientes al $54.3 \%$ del total) reconocen que la principal está relacionada con los horarios, ya que al tratarse de una herramienta sincrónica exige el establecimiento de unos horarios de atención específicos, que en algunas ocasiones coinciden con otras actividades laborales o personales de los estudiantes. Entre otras desventajas se mencionan: la dificultad

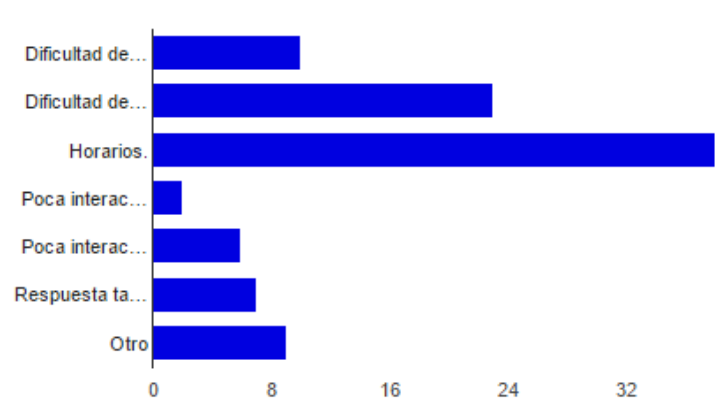

de conexión (23 estudiantes, correspondientes al $32.9 \%$ del total) $y$, en menor medida, la dificultad de uso de esta herramienta en línea (10 estudiantes, correspondientes al $14.3 \%$ del total) (Figura 16). Esta herramienta es muy útil en los procesos formativos, ya que permite el contacto directo de docentes y estudiantes, facilitando así el intercambio de ideas y la respuesta inmediata a las diferentes inquietudes o consultas.

Figura 18. Desventajas del uso del Chat en línea.

\section{Conclusiones}

En los cursos virtuales se evidencia un amplio uso de las diferentes herramientas de comunicación para el proceso de aprendizaje, observándose mayor preferencia por las asincrónicas frente a las sincrónicas, principalmente por la facilidad que ofrecen aquellas para participar en cualquier momento, situación que limita a las segundas por la necesidad de tener un horario específico para la interacción y participación. Sin embargo, las dos ofrecen amplias posibilidades de intercambio de ideas y opiniones, y dan lugar a procesos de construcción del conocimiento; aquí es donde el docente debe ser un motivador y elemento integrador para que el estudiante pueda dar un uso amplio y pertinente a estas herramientas para el logro de los objetivos, propósitos y metas de formación establecidas en cada uno de los cursos. 
Las herramientas sincrónicas (Skype, chat en línea, telefonía fija o celular) son utilizadas en menor medida, a pesar de que existe una percepción en los estudiantes acerca de que estas facilitan mayor interacción con el docente y los otros compañeros; su principal limitante se encuentra en la exigencia de un horario específico de conexión; igualmente, en algunos casos la velocidad de conexión se puede constituir también en un factor limitante para el uso de estas herramientas de tipo sincrónico.

Las herramientas de tipo asincrónico se utilizan en mayor medida, principalmente, por la posibilidad de plantear las ideas y opiniones en cualquier momento, sin necesidad de contar con un horario específico, ya que la interacción se desarrolla en diferentes tiempos. Sin embargo, debe propugnarse un mayor uso para la interacción de los estudiantes, de forma tal que puedan articularse de manera exitosa con la estrategia de aprendizaje planteada por el docente.

Para el contacto con el docente, los estudiantes prefieren el correo interno de los cursos, los foros de contacto y los foros de trabajo; sin embargo, los encuestados evidencian el interés por utilizar en mayor medida las herramientas sincrónicas, ya que encuentran como principal ventaja la mejor interacción que estas pueden brindar tanto con el docente como con los otros estudiantes, al permitir una comunicación directa, bidireccional y en tiempo real.

En el contacto con los otros estudiantes se prefiere el uso de los foros de trabajo y del teléfono celular, y en menor medida el correo interno de los cursos. Se evidencia una baja utilización de las diversas herramientas disponibles y se debe motivar la utilización de estas y el desarrollo de procesos más participativos e interactivos. En este sentido, las interacciones están enfocadas principalmente en el desarrollo de las actividades colaborativas y de tipo práctico de los cursos.

Se deben implementar acciones tendientes a incrementar el uso de las herramientas sincrónicas, con el fin de facilitar la comunicación inmediata en los cursos virtuales. En este mismo sentido, se deben brindar espacios de atención más amplios, con el fin de facilitar la utilización de estas herramientas por los estudiantes.

Uno de los aspectos que deben tenerse en cuenta en el uso de estas herramientas de comunicación en los procesos de formación en ambientes virtuales de aprendizaje es su función pedagógica, por lo cual deben estar enmarcadas en la estrategia de aprendizaje que se desarrolle en los cursos; esto con el fin de mejorar su utilización y aprovechamiento en la consecución de los objetivos, metas y propósitos de formación propuestos. Cada herramienta debe tener un papel específico en el curso; sin embargo, debe brindarse la libertad de elección de utilización por los estudiantes, ya que ellos son los artífices de su propio conocimiento y, en ese sentido, son quienes establecen y articulan los diferentes procesos y herramientas para la generación de procesos formativos pertinentes al logro de sus metas de formación.

\section{Referencias}

(1) Artal Sevil JS, Caraballo Jiménez J., Artacho Terrer JM. Uso del google-talk y skype como herramienta interactiva en la docencia semipresencial. España: Universidad de Zaragoza. Sf.

(2) García Aretio L. Blended Learning. ¿Enseñanza y Aprendizaje integrados? España: Editorial del BENED. 2004.

(3) Cabero Almenara J., Llorente Cejudo MC. La interacción en el aprendizaje en red: uso de herramientas, elementos de análisis y posibilidades educativas. RIED, 10(2): 97-123, 2007.

(4) Sánchez Martínez F., Prendes Espinosa MP. Estrategias y espacios virtuales de colaboración para la enseñanza superior. Revista Internacional de Ciencias Sociales y Humanidades. SOCIOTAM. Universidad Autónoma de Tamaulipas. Ciudad Victoria, México. XVIII (2): 59-90, 2008.

(5) Grupo del Banco Mundial. Indicadores de población rural. 2015. 
(6) Daza MM, Tapia MM, Chiarani MC. Los foros virtuales como herramienta pedagógico-didáctica en la evaluación de procesos de aprendizaje. Argentina: Universidad Nacional de San Luis. Sf.

(7) Hernández MA. Estos son los indicadores TIC en los que vamos 'rajados'. Diario El Tiempo. 30 de marzo, 2015.

(8) Arnau C. Skype en la educación. Barcelona: Universidad Autónoma de Barcelona. 2009.
(9) Verdugo Valcarce A. Influencia del uso de herramientas técnicas sincrónicas en la enseñanza-aprendizaje de lenguas extranjeras: inglés para turismo. Facultad de Filología. Departamento de Filologías Extranjeras y sus Lingüísticas. Madrid. 2008.

(10) Valverde Berrocoso J., Garrido Arroyo MC. La función tutorial en entornos virtuales de aprendizaje. Revista Latinoamericana de Tecnología Educativa, 4(1): 153-167, 2005. 